\title{
Linkage and Association Study of Neurotrophins and Their Receptors as Novel Susceptibility Genes for Childhood IgA Nephropathy
}

\author{
WON-HO HAHN, JIN-SOON SUH, AND BYOUNG-SOO CHO
}

Department of Pediatrics, School of Medicine, Kyung Hee University, Seoul 130-702, Republic of Korea

\begin{abstract}
Neurotrophins (NTs) and their receptors (NTRs) are known to be important for pathogenesis of various inflammatory diseases that occur in not only neuronal but also nonneuronal tissues, including kidney. Here, we investigated association between childhood IgA nephropathy (IgAN) and single nucleotide polymorphisms (SNPs) of genes encoding NTs [nerve growth factor (NGF) and brain-derived neurotrophic factor (BDNF)] and NTRs [nerve growth factor receptor (NGFR) and neurotrophic tyrosine kinase receptor 1-3 (NTRK1-3)]. The genotyping data of 197 patients and 289 control subjects revealed significant association between NGF SNP rs11102930 and presence of IgAN. Patient subgroup analysis revealed that that the presence of nephrotic range proteinuria $(>40$ $\mathrm{mg} / \mathrm{m}^{2} / \mathrm{h}$ ) was associated with rs6334 of NTRK1 and rs11030104, rs7103411, rs7103873, and rs6484320 of BDNF. Significant genotype differences were observed in podocyte foot process effacement for rs1187321 and rs1187323 of NTRK2. Furthermore, some SNPs showed significantly different genotype distribution between patients with or without pathologically advanced disease markers, specifically in rs6334 of NTRK1. Our results suggest that SNPs of NTs and NTRs are associated with susceptibility, pathological advancement, podocyte foot process effacement, and development of proteinuria in childhood IgAN. (Pediatr Res 69: 299-305, 2011)
\end{abstract}

I gA nephropathy (IgAN) is the most commonly occurring form of chronic glomerulonephritis (GN) in pediatric patients and is defined by $\operatorname{IgA}$ deposition in the glomerular mesangium accompanied by a mesangial proliferative GN. The extent and intensity of glomerular injury in response to mesangial IgA deposition are extremely variable and determine the levels of subsequent mesangial cell proliferation and interstitial fibrosis. Polymorphisms of candidate genes that are involved in these processes could thus affect disease susceptibility and progression.

The neurotrophins (NTs) are a family of neurotrophic factors that are essential for the development of the nervous system, regulating the survival, death, tissue repair, and differentiation of neurons (1). There are four NT family members: nerve growth factor (NGF), brain-derived neurotrophic factor (BDNF), neurotrophin-3 (NT-3), and neurotrophin-4/5 $($ NT-4/5) (1). The biological effects of all NTs are mediated by

Received March 8 2010; accepted October 28, 2010.

Correspondence: Byoung-Soo Cho, M.D., Ph.D., Department of Pediatrics, School of Medicine, Kyung Hee University, Hoegi-dong \#1, Dongdaemun-gu, Seoul 130-702, Republic of Korea; e-mail: bscho@dreamwiz.com

Supported by the 2009 Young Researcher of Medical Science Program at Kyung Hee University (KHU-20091437). neurotrophin receptors (NTRs), which include tyrosine kinase receptors (TrkA, B, and $\mathrm{C}$ ) and nerve growth factor receptor (NGFR, also known as NT receptor p75). NTs bind to a common family of Trk cell surface receptors with high affinity and variable specificity. NGF binds preferentially to TrkA (encoded by NTRK1), BDNF and NT-4/5 bind to TrkB (encoded by NTRK2), and NT-3 binds to TrkC (encoded by NTRK3). All NTs also bind to the common low-affinity NT receptor NGFR (encoded by NGFR) (1). Signaling through Trk receptors includes different pathways, such as the Ras/ ERK pathway, the phosphatidylinositol-3-kinase (PI3K)/Akt kinase pathway, the phospholipase $\mathrm{C} \gamma$ (PLC $\gamma$ ) pathway, and the Smad transduction pathway $(1,2)$. The NGFR-mediated signaling is more complex, involving both Trk-dependent and -independent signal transductions.

NTs have been studied mainly in relation to neurological and psychological diseases. However, increasing evidences indicate that NTs and NTRs are implicated in the pathogenesis of various inflammatory diseases (3). Moreover, NTs were found to be expressed in the kidney tissues, and it has been suggested that the NTs has an enough possibility to be involved in the pathophysiology of renal disease, including IgAN.

There have been several single nucleotide polymorphisms (SNPs) studies on neuronal and nonneuronal tissue-associated diseases (4-7). However, there have been no studies linking renal diseases with SNPs of genes encoding NT family members and their receptors, especially in pediatric IgAN. Here, we have investigated associations between polymorphisms of NT pathway genes and childhood IgAN.

\section{METHODS}

Patients and controls. We examined a total of 197 Korean pediatric patients with IgAN confirmed by biopsy (mean age $\pm \mathrm{SD}, 12.63 \pm 5.19 \mathrm{y}$; 117 boys, $13.39 \pm 4.98 \mathrm{y} ; 80$ girls, $11.53 \pm 5.31 \mathrm{y}$ ) and compared them with 289 healthy control subjects (mean age \pm SD, $37.63 \pm 13.35$ y; 157 males, $39.63 \pm 14.72 \mathrm{y} ; 132$ females, $35.25 \pm 11.11$ y). The follow-up duration before the renal biopsy was $21.7 \pm 27.8 \mathrm{mo}$. Patients were detected through abnormal urinalysis results during school screenings, and most of them showed no other symptoms of GN. Thus, they were assumed to have
Abbreviations: BDNF, brain-derived neurotrophic factor; GN, glomerulonephritis; IgAN, IgA nephropathy; LD, linkage disequilibrium; NGF (R), nerve growth factor (receptor); NT (R), neurotrophin (receptor); NTF-3, neurotrophin-3; NTRK, neurotrophic tyrosine kinase receptor; SNP, single nucleotide polymorphism; Trk, tyrosine kinase receptor 
relatively early stage disease. At our center, we performed a renal biopsy in all patients with i) unexplained prolonged isolated hematuria or proteinuria of duration $\geq 12 \mathrm{mo}$, ii) concomitant hematuria and proteinuria for $>3 \mathrm{mo}$, iii) a second episode of gross hematuria with decreased serum $\mathrm{C} 3$ and $\mathrm{C} 4$ levels, or iv) decreased renal function. Healthy control subjects were also recruited based on routine screenings. This screening included the completion of a questionnaire addressing the presence of symptoms, medical history, measurement of blood pressure, electrocardiography, abdominal sonography, and laboratory tests such as complete blood count, fasting glucose level, total cholesterol, triglyceride, HDL-cholesterol, rheumatoid factor, hepatitis viral markers, Hb A1C, liver enzymes, blood urea nitrogen, creatinine, electrolytes, and urinalysis findings (protein, glucose, and occult blood). Control candidates with an abnormal result for any item were excluded.

Patient subgroups. To determine the natures of the associations between SNPs of NTs and NTRs and the development of proteinuria, we divided patients into subgroups according to the largest amount of proteinuria observed during the course of disease (nephrotic range proteinuria $>40$ and $\leq 40$ $\mathrm{mg} / \mathrm{m}^{2} / \mathrm{h}$ ).

In addition, patients were allocated to gross hematuria $(+)$ and $(-)$ groups according to the presence of gross hematuria episodes as an initial symptom of IgAN. Patients with IgAN were also divided into mild and advanced disease subgroups to evaluate the contribution of the SNPs in disease progression in terms of pathologic findings. Members of the advanced disease group had at least one of the following pathological markers: interstitial fibrosis, tubular atrophy, or global sclerosis. Finally, we further divided patients with $\operatorname{IgAN}$ into podocyte foot process effacement $(+)$ and $(-)$ subgroups according to renal biopsy. The demographic characteristics of patients with IgAN were summarized, with small differences in subgroup numbers resulting from the loss of some clinical data (Table 1).

This study was approved by the ethics review committee of the Medical Research Institute, Kyung Hee University Medical Center, Seoul. Written informed consent was obtained from all subjects and from the parents or legal guardians.

SNP selection and genotyping. Three SNPs of $N G F$, one SNP on NGFR, four SNPs of $B D N F$, four SNPs of NTRK1, two SNPs of NTRK2, and one SNP of NTRK3 were selected based on the findings of extensive database searches (http://www.ebi.ac.uk/ensemble/ and http://ncbi.nlm.nih.gov/SNP) for heterozygosity $>0.1$ and a minor allele frequency (MAF) of $>0.05$ (Table 2).

DNA was isolated from peripheral blood samples using Core One Blood Genomic DNA Isolation Kits (CoreBioSystem, Seoul). SNP genotyping was conducted by direct sequencing. Genomic DNA was amplified using specific primers for the 15 SNPs of NTs and their receptor genes. The samples were sequenced using an ABI Prism 377 automatic sequencer (PE Applied Biosystems, Foster City, CA), and the sequence data were analyzed using SeqManII software (DNASTAR Inc., Madison, WI).

Statistical analysis. We analyzed 15 SNPs of genes encoding NTs and their receptors in the 197 patients and 289 controls. For the case-control association study, Hardy-Weinberg equilibrium (HWE) for all SNPs was assessed using SNPstats (Biostatistics and Bioinformatics Unit, Barcelona, Spain) in both cases and controls (8), and the SNPs not in HWE $(p<0.05)$ were excluded from the analysis. For the logistic regression analysis and trend test, we used SNPstats and SNPAnalyzer (ISTECH Inc., Goyang, Korea). To show alternative effects of the variants, logistic regression analysis was performed in the statistical genetic models (codominant, dominant, recessive, and overdominant models) (9). For the SNPs that do not have all three genotypes present in the study population, only allele frequencies were compared by the $\chi^{2}$ test. And they were taken into account of Bonferroni's correction. To reduce experimental error, we calculated sample power for the SNPs and the number of cases was adjusted to achieve $80 \%$ power $(\alpha=0.05$, genotype relative risk $=2$-fold $)$ using a genetic power calculator (http://pngu.mgh.harvard.edu/ purcell/gpc).

A linkage disequilibrium (LD) block of polymorphisms and haplotype analysis was tested using Haploview version 4.2 (Broad Institute, Cambridge, MA), HapAnalyzer version 1.0 (http://hap.ngri.go.kr/), and HelixTree (Golden Helix Inc., Bozeman, MT). We examined Lewontin's $D^{\prime}$ and $r^{2}$ between all pairs of biallelic loci (10). The haplotypes and their frequencies were inferred using the expectation-maximization (EM) algorithm (11).

\section{RESULTS}

Despite the fact that most patients were asymptomatic at presentation, analysis of the subgroups of patients with IgAN showed that the presence of podocyte effacement were correlated with the advanced pathologic classification of IgAN according to the H. S. Lee's glomerular grading system (12) (Table 1).

The genotypic distributions of all SNPs in this study were consistent with Hardy-Weinberg equilibrium $(p>0.05)$. The genotyping data of 197 patients and 289 controls revealed a significant association between $N G F$ rs11102930 and the presence of IgAN by logistic regression analysis after adjusting for gender (codominant model: $\mathrm{OR}=0.67,95 \% \mathrm{CI}=0.51-0.88$, $p=0.003$; dominant model: $\mathrm{OR}=0.62,95 \% \mathrm{CI}=0.42$ $0.91, p=0.015$; recessive model: $\mathrm{OR}=0.56,95 \% \mathrm{CI}=$ $0.34-0.90, p=0.015$; Table 3 ). We calculated sample power for this SNP, rs11102930. In our case-control study, we had 0.97 for codominant model [effective sample size $(\mathrm{ESS})=$ 239], 0.98 for dominant model ( $E S S=152), 0.53$ for recessive model $(\mathrm{ESS}=163)$ power to detect a 2 -fold increased risk, assuming an $\alpha$-level of 0.05 . Thus, codominant and

Table 1. Demographics of the patients with IgA nephropathy $(n=197)$

\begin{tabular}{|c|c|c|c|c|c|c|c|c|c|c|c|}
\hline \multirow[b]{2}{*}{ Subgroup } & \multirow[b]{2}{*}{$n(\%)$} & \multirow[b]{2}{*}{$\mathrm{M}: \mathrm{F}$} & \multirow{2}{*}{$\begin{array}{c}\text { Age } \\
(\mathrm{y} ; \text { mean } \pm \mathrm{SD})\end{array}$} & \multirow[b]{2}{*}{ Mean } & \multirow{2}{*}{$\begin{array}{l}\text { Follow-up } \\
\text { duration before } \\
\text { renal biopsy } \\
\text { (months) }\end{array}$} & \multicolumn{6}{|c|}{ Pathologic Grading $(n)^{*}$} \\
\hline & & & & & & I & II & III & IV & $\mathrm{V}$ & $p$ \\
\hline \multicolumn{12}{|l|}{ Proteinuria $\left(\mathrm{mg} / \mathrm{m}^{2} / \mathrm{h}\right) \dagger$} \\
\hline$>40$ & $33(16.8)$ & $23: 10$ & $11.41 \pm 4.76$ & $104.48 \pm 78.77$ & $21.7 \pm 27.7$ & 17 & 16 & 0 & 0 & 0 & 0.072 \\
\hline$\leq 40$ & $164(83.2)$ & $94: 70$ & $12.84 \pm 5.22$ & $10.71 \pm 9.68$ & $21.8 \pm 27.8$ & 115 & 48 & 0 & 1 & 0 & \\
\hline \multicolumn{12}{|l|}{ Gross hematuria } \\
\hline+ & $37(18.8)$ & $21: 16$ & $12.88 \pm 7.68$ & NA§ & $21.9 \pm 28.1$ & 24 & 12 & 0 & 1 & 0 & 0.384 \\
\hline- & $160(81.2)$ & $96: 64$ & $12.53 \pm 4.41$ & $\mathrm{NA}$ & $21.7 \pm 27.8$ & 108 & 52 & 0 & 0 & 0 & \\
\hline \multicolumn{12}{|c|}{ Advanced disease markers } \\
\hline+ & $21(10.7)$ & $16: 05$ & $15.71 \pm 6.72$ & NA & $21.5 \pm 27.6$ & 6 & 14 & 0 & 1 & 0 & $<0.001$ \\
\hline- & $176(89.3)$ & $101: 75$ & $12.22 \pm 4.83$ & NA & $21.7 \pm 27.8$ & 126 & 50 & 0 & 0 & 0 & \\
\hline \multicolumn{12}{|c|}{ Podocyte foot process effacement } \\
\hline+ & $72(36.5)$ & $44: 28$ & $12.80 \pm 5.34$ & NA & $21.6 \pm 27.7$ & 33 & 38 & 0 & 1 & 0 & $<0.001$ \\
\hline- & $125(63.5)$ & $73: 52$ & $12.48 \pm 5.07$ & NA & $21.6 \pm 27.8$ & 99 & 26 & 0 & 0 & 0 & \\
\hline
\end{tabular}

*Modified H. S. Lee's glomerular grading system (12); Chi-square test was performed to determine the correlation between pathologic grading and the presence of proteinuria, gross hematuria, advanced disease markers, and podocyte foot process effacement.

$\dagger$ Proteinuria indicates the largest level of proteinuria observed during the course of disease.

\$ross hematuria developed as a first symptom of IgA nephropathy.

$\S N A$, not applicable. 
Table 2. Information and sequences of primers of candidate SNPs of neurotrophins and their receptor-encoding genes

\begin{tabular}{|c|c|c|c|c|c|c|}
\hline \multirow[b]{2}{*}{ Gene } & \multirow[b]{2}{*}{ SNPs } & \multirow[b]{2}{*}{ Function } & \multicolumn{4}{|c|}{ Primers and conditions } \\
\hline & & & & Sequence $\left(5^{\prime}-3^{\prime}\right)$ & Product size $(b p)$ & Annealing temperature $\left({ }^{\circ} \mathrm{C}\right)$ \\
\hline \multirow[t]{6}{*}{$N G F(1 \mathrm{p} 13)$} & \multirow[t]{2}{*}{ rs6330 } & \multirow[t]{2}{*}{ Ala273Val } & Forward & CATTCCAGGTGCATAGCGTAAT & \multirow[t]{2}{*}{328} & \multirow[t]{2}{*}{65} \\
\hline & & & Reverse & AGATCCTGAGTGTCTGCAGCTT & & \\
\hline & \multirow[t]{2}{*}{ rs 3738701} & \multirow[t]{2}{*}{ Intron } & Forward & GGAGAGGTGAGGGAAGCTG & \multirow[t]{2}{*}{321} & \multirow{2}{*}{65} \\
\hline & & & Reverse & CTCCCGTACTCCTGAGTCACAC & & \\
\hline & \multirow[t]{2}{*}{ rs11102930 } & \multirow[t]{2}{*}{ Promoter } & Forward & AACAGTTTTACCAAGGGAGCAG & \multirow[t]{2}{*}{355} & \multirow[t]{2}{*}{62} \\
\hline & & & Reverse & GAGTTGTGTGGAGGGTCTGACT & & \\
\hline \multirow[t]{8}{*}{ NTRK1 (1p21) } & \multirow[t]{2}{*}{ rs926103 } & \multirow[t]{2}{*}{ Promoter } & Forward & СTTCATCTATTCCTGGCAGGTG & \multirow[t]{2}{*}{381} & \multirow[t]{2}{*}{59} \\
\hline & & & Reverse & TAGACAGGAGAGGCTGGGTATG & & \\
\hline & \multirow[t]{2}{*}{ rs2150906 } & \multirow[t]{2}{*}{ Promoter } & Forward & TCCAGAGACTCTCCTTCCTTTG & \multirow[t]{2}{*}{312} & \multirow[t]{2}{*}{62} \\
\hline & & & Reverse & GCACTCTGTGTCAGTGACCATT & & \\
\hline & \multirow[t]{2}{*}{ rs1800601 } & \multirow[t]{2}{*}{$5^{\prime} \mathrm{UTR}$} & Forward & AAATTATTGACTGGGCAGGAGA & \multirow[t]{2}{*}{301} & \multirow[t]{2}{*}{62} \\
\hline & & & Reverse & AAGGACTTGCAGATGGACAAAG & & \\
\hline & rs6334 & Synonymous & Forward & CTCCATCACATCAGGACAGAGT & 382 & 62 \\
\hline & & & Reverse & TGTCTATAGGGAAGGGAAGACG & & \\
\hline NTRK2 $(9 \mathrm{q} 22)$ & rs1187321 & Promoter & Forward & ACCACTCGATGTGTGTTACAGC & 304 & 58 \\
\hline & & & Reverse & AGAGAGCAATGGGTTGGAGTCT & & \\
\hline & rs1187323 & Promoter & Forward & GCGCATCTGGCGCCAGAGCGCG & 425 & 65 \\
\hline & & & Reverse & GTGTTCATGTGTGCTAGGGTGT & & \\
\hline$B D N F(11 \mathrm{p} 13)$ & rs11030104 & Intron & Forward & AGAGTCATCCGAAGGTTGAAAA & 353 & 58 \\
\hline & & & Reverse & TGCGGATCCCTGCTCTAAGGAA & & \\
\hline & rs7103411 & Intron & Forward & TGCATCTGATTTCAGAGGTGAG & 387 & 62 \\
\hline & & & Reverse & AAACATAGGAGGGGAAAAGGAC & & \\
\hline & rs7103873 & Intron & Forward & GGGAAGTCTTGAATTTTTGTGC & 377 & 62 \\
\hline & & & Reverse & GGGAGCGCACTGTAAAGATACT & & \\
\hline & rs6484320 & Intron & Forward & GCAGTGCTTGGCATAGTAAATG & 334 & 59 \\
\hline & & & Reverse & AGGAACTCAGATAGGGCAGGTT & & \\
\hline NTRK3 $(15 \mathrm{q} 25)$ & rs1128994 & Synonymous & Forward & TCTTCGGTTCAGAGGTTCCCTT & 418 & 65 \\
\hline & & & Reverse & CCTGAAACCAGTCTTCCTATGG & & \\
\hline$N G F R(17 \mathrm{q} 21)$ & rs11466155 & Synonymous & Forward & GTTGGATTACACGGTCCACACC & 376 & 59 \\
\hline & & & Reverse & GCAGATGATGAGTGAGGATGAG & & \\
\hline
\end{tabular}

dominant model of rs11102930 were sufficiently powerful to determine positive association.

When we assessed genetic association between the 15 SNPs and subgroups of patients with IgAN, NGF rs6330 was found to be associated with the presence of gross hematuria as an initial IgAN symptom (dominant model: OR $=2.25,95 \%$ $\mathrm{CI}=1.06-4.80, p=0.036$; overdominant model: OR = $2.27,95 \% \mathrm{CI}=1.06-4.88, p=0.036)$. In addition, the presence of nephrotic range proteinuria was found to be associated with NTRK1 rs6334 (overdominant model: OR = $2.40,95 \% \mathrm{CI}=1.09-5.28, p=0.026), B D N F$ rs11030104 (codominant model: $\mathrm{OR}=0.49,95 \% \mathrm{CI}=0.28-0.86, p=$ 0.010 ; recessive model: $\mathrm{OR}=0.14,95 \% \mathrm{CI}=0.03-0.63$, $p=0.001$ ), BDNF rs7103411 (codominant model: OR = $0.49,95 \%$ CI $=0.28-0.86, p=0.010$; recessive model: $\mathrm{OR}=0.14,95 \% \mathrm{CI}=0.03-0.63, p=0.001), B D N F$ rs7103873 (dominant model: $\mathrm{OR}=3.56,95 \% \mathrm{CI}=1.17-$ $10.79, p=0.012$ ), and BDNF rs6484320 (codominant model: $\mathrm{OR}=0.49,95 \% \mathrm{CI}=0.28-0.86, p=0.010$; recessive model: $\mathrm{OR}=0.14,95 \% \mathrm{CI}=0.03-0.63, p=0.001$; Table 4).

In terms of podocyte foot process effacement, NTRK2 rs1187321 (recessive model: $\mathrm{OR}=5.82,95 \% \mathrm{CI}=1.13$ 29.89, $p=0.021$ ) and NTRK2 rs1187323 (recessive model: $\mathrm{OR}=5.82,95 \% \mathrm{CI}=1.13-29.89, p=0.021)$ showed significant differences in genotype distribution (data not shown).

Comparison of genotype differences between the advanced pathologic marker $(+)$ and $(-)$ groups revealed a significant association with NTRK1 rs6334 (overdominant model: OR = $3.19,95 \% \mathrm{CI}=1.16-8.75, p=0.018$; data not shown).

In measurements of pair-wise LD, one LD block was identified in the SNPs of NTRK1 (composed with rs926103, rs2150906, and rs1800601) by the Gabriel method (13), but it was not statistically significant (data not shown).

\section{DISCUSSION}

NTs have been studied mainly in relation to neurological and psychological diseases (1). However, there is increasing evidence that NTs and NTRs are also expressed in nonneuronal tissues $(1,3)$, and several recent works have elucidated a regulatory effect of NTs on the proinflammatory mediators, such as IL- $1 \beta$, TNF- $\alpha$, and IL-6 $(3,14)$. In addition, BDNF and NGF are detectable and over-expressed in the synovial tissues and fluids of patients with rheumatoid arthritis (RA) and spondyloarthritis but not in healthy controls (15-17). Additional evidence exists for an association between increased levels of NTs and susceptibility to inflammatory diseases such as systemic lupus erythematosus (SLE) $(18,19)$, RA, multiple sclerosis, and juvenile chronic arthritis (20-22). Even a Disease Activity Index in patients with SLE was significantly correlated with serum levels of NGF (19).

In the kidney, the NTs and their receptors are expressed in both fetal and adult renal tissues; renal tubular, interstitial, and glomerular cells, and especially mesangial cells and podocytes (23-28). In addition, NT systems are critical for kidney de- 
Table 3. Logistic regression analysis of neurotrophins and their receptor-encoding gene polymorphisms in IgA nephropathy patients and healthy controls after adjustment for age*

\begin{tabular}{|c|c|c|c|c|c|c|c|}
\hline Gene symbol & SNP & Genotype & $\begin{array}{c}\text { Controls } \\
(n=289 ; \%)\end{array}$ & $\begin{array}{c}\operatorname{IgAN} \\
(n=197 ; \%)\end{array}$ & Model & OR $(95 \% \mathrm{CI})$ & $p$ \\
\hline \multirow[t]{12}{*}{$N G F$} & rs6330 & $\mathrm{C} / \mathrm{C}$ & $182(63)$ & $130(66.7)$ & Codominant & $0.92(0.65-1.31)$ & 0.640 \\
\hline & Ala273Val & $\mathrm{T} / \mathrm{C}$ & $104(36)$ & $60(30.8)$ & Dominant & $0.85(0.58-1.25)$ & 0.400 \\
\hline & & $\mathrm{T} / \mathrm{T}$ & $3(1)$ & $5(2.6)$ & Recessive & $2.42(0.57-10.27)$ & 0.220 \\
\hline & & & & & Overdominant & $0.79(0.54-1.17)$ & 0.240 \\
\hline & rs3738701 & $\mathrm{C} / \mathrm{C}$ & $241(83.7)$ & $173(87.8)$ & Codominant & $0.73(0.44-1.22)$ & 0.230 \\
\hline & intron & $\mathrm{A} / \mathrm{C}$ & $46(16)$ & $23(11.7)$ & Dominant & $0.70(0.41-1.19)$ & 0.190 \\
\hline & & $\mathrm{A} / \mathrm{A}$ & $1(0.4)$ & $1(0.5)$ & Recessive & $1.66(0.10-26.89)$ & 0.720 \\
\hline & & & & & Overdominant & $0.68(0.40-1.17)$ & 0.160 \\
\hline & rs11102930 & $\mathrm{C} / \mathrm{C}$ & $76(26.3)$ & $71(36.6)$ & Codominant & $0.67(0.51-0.88)$ & 0.003 \\
\hline & Promoter & $\mathrm{T} / \mathrm{C}$ & $146(50.5)$ & 95 (49) & Dominant & $0.62(0.42-0.91)$ & 0.015 \\
\hline & & $\mathrm{T} / \mathrm{T}$ & $67(23.2)$ & $28(14.4)$ & Recessive & $0.56(0.34-0.90)$ & 0.015 \\
\hline & & & & & Overdominant & $0.94(0.65-1.35)$ & 0.740 \\
\hline \multirow[t]{16}{*}{ NTRK1 } & rs926103 & $\mathrm{G} / \mathrm{G}$ & $198(68.8)$ & $137(69.5)$ & Codominant & $0.95(0.67-1.34)$ & 0.770 \\
\hline & Promoter & $\mathrm{A} / \mathrm{G}$ & $82(28.5)$ & 55 (27.9) & Dominant & $0.95(0.64-1.41)$ & 0.790 \\
\hline & & $\mathrm{A} / \mathrm{A}$ & $8(2.8)$ & $5(2.5)$ & Recessive & $0.90(0.29-2.80)$ & 0.860 \\
\hline & & & & & Overdominant & $0.96(0.64-1.44)$ & 0.830 \\
\hline & rs2150906 & $\mathrm{G} / \mathrm{G}$ & $250(87.4)$ & $174(88.8)$ & Codominant & $0.84(0.48-1.46)$ & 0.530 \\
\hline & Promoter & $\mathrm{A} / \mathrm{G}$ & $35(12.2)$ & $22(11.2)$ & Dominant & $0.86(0.49-1.51)$ & 0.600 \\
\hline & & $\mathrm{A} / \mathrm{A}$ & $1(0.4)$ & $0(0)$ & Recessive & 0.00 (0.00-NA) & 0.290 \\
\hline & & & & & Overdominant & $0.89(0.50-1.57)$ & 0.680 \\
\hline & rs1800601 & $\mathrm{T} / \mathrm{T}$ & $198(68.8)$ & $137(69.5)$ & Codominant & $0.95(0.67-1.34)$ & 0.770 \\
\hline & 5'UTR & $\mathrm{T} / \mathrm{C}$ & $82(28.5)$ & $55(27.9)$ & Dominant & $0.95(0.64-1.40)$ & 0.780 \\
\hline & & $\mathrm{C} / \mathrm{C}$ & $8(2.8)$ & $5(2.5)$ & Recessive & $0.90(0.29-2.80)$ & 0.860 \\
\hline & & & & & Overdominant & $0.96(0.64-1.43)$ & 0.830 \\
\hline & rs6334 & $\mathrm{G} / \mathrm{G}$ & $111(38.7)$ & $81(41.1)$ & Codominant & $0.94(0.72-1.24)$ & 0.660 \\
\hline & Synonymous & $\mathrm{A} / \mathrm{G}$ & $141(49.1)$ & $92(46.7)$ & Dominant & $0.89(0.61-1.29)$ & 0.540 \\
\hline & & $\mathrm{A} / \mathrm{A}$ & $35(12.2)$ & $24(12.2)$ & Recessive & $1.01(0.58-1.76)$ & 0.980 \\
\hline & & & & & Overdominant & $0.89(0.62-1.28)$ & 0.530 \\
\hline \multirow[t]{8}{*}{ NTRK2 } & rs1187321 & $\mathrm{A} / \mathrm{A}$ & $164(56.8)$ & $118(59.9)$ & Codominant & $0.87(0.64-1.19)$ & 0.390 \\
\hline & Promoter & $\mathrm{A} / \mathrm{T}$ & 109 (37.7) & $71(36)$ & Dominant & $0.88(0.61-1.26)$ & 0.480 \\
\hline & & $\mathrm{T} / \mathrm{T}$ & $16(5.5)$ & $8(4.1)$ & Recessive & $0.73(0.30-1.73)$ & 0.460 \\
\hline & & & & & Overdominant & $0.93(0.64-1.35)$ & 0.690 \\
\hline & rs1187323 & $\mathrm{A} / \mathrm{A}$ & $165(57.1)$ & 118 (59.9) & Codominant & $0.88(0.65-1.20)$ & 0.430 \\
\hline & Promoter & $\mathrm{A} / \mathrm{C}$ & 108 (37.4) & $71(36)$ & Dominant & $0.89(0.61-1.28)$ & 0.530 \\
\hline & & $\mathrm{C} / \mathrm{C}$ & $16(5.5)$ & $8(4.1)$ & Recessive & $0.73(0.31-1.75)$ & 0.480 \\
\hline & & & & & Overdominant & $0.94(0.64-1.37)$ & 0.740 \\
\hline \multirow[t]{16}{*}{$B D N F$} & rs11030104 & $\mathrm{A} / \mathrm{A}$ & 77 (26.6) & $51(25.9)$ & Codominant & $1.12(0.86-1.46)$ & 0.380 \\
\hline & Intron & $\mathrm{A} / \mathrm{G}$ & 155 (53.6) & $97(49.2)$ & Dominant & $1.03(0.68-1.56)$ & 0.880 \\
\hline & & $\mathrm{G} / \mathrm{G}$ & $57(19.7)$ & $49(24.9)$ & Recessive & $1.33(0.86-2.06)$ & 0.200 \\
\hline & & & & & Overdominant & $0.84(0.59-1.21)$ & 0.350 \\
\hline & rs7103411 & $\mathrm{T} / \mathrm{T}$ & $79(27.3)$ & $51(25.9)$ & Codominant & $1.14(0.88-1.48)$ & 0.330 \\
\hline & Intron & $\mathrm{T} / \mathrm{C}$ & $153(52.9)$ & $97(49.2)$ & Dominant & $1.07(0.71-1.61)$ & 0.750 \\
\hline & & $\mathrm{C} / \mathrm{C}$ & $57(19.7)$ & 49 (24.9) & Recessive & $1.33(0.86-2.06)$ & 0.200 \\
\hline & & & & & Overdominant & $0.86(0.60-1.24)$ & 0.430 \\
\hline & rs7103873 & $\mathrm{G} / \mathrm{G}$ & $75(25.9)$ & $56(28.4)$ & Codominant & $0.96(0.74-1.25)$ & 0.770 \\
\hline & Intron & $\mathrm{G} / \mathrm{C}$ & $155(53.6)$ & $100(50.8)$ & Dominant & $0.89(0.59-1.33)$ & 0.570 \\
\hline & & $\mathrm{C} / \mathrm{C}$ & $59(20.4)$ & $41(20.8)$ & Recessive & $1.03(0.66-1.61)$ & 0.900 \\
\hline & & & & & Overdominant & $0.89(0.62-1.28)$ & 0.540 \\
\hline & rs6484320 & $\mathrm{A} / \mathrm{A}$ & $80(27.7)$ & $51(25.9)$ & Codominant & $1.15(0.88-1.49)$ & 0.310 \\
\hline & Intron & T/A & $152(52.6)$ & $97(49.2)$ & Dominant & $1.09(0.72-1.64)$ & 0.690 \\
\hline & & $\mathrm{T} / \mathrm{T}$ & $57(19.7)$ & $49(24.9)$ & Recessive & $1.33(0.86-2.06)$ & 0.200 \\
\hline & & & & & Overdominant & $0.88(0.61-1.26)$ & 0.470 \\
\hline \multirow[t]{4}{*}{ NTRK3 } & rs1128994 & $\mathrm{C} / \mathrm{C}$ & $185(64.5)$ & $124(63.6)$ & Codominant & $1.08(0.77-1.51)$ & 0.650 \\
\hline & Synonymous & $\mathrm{T} / \mathrm{C}$ & $96(33.5)$ & $64(32.8)$ & Dominant & $1.04(0.71-1.52)$ & 0.830 \\
\hline & & $\mathrm{T} / \mathrm{T}$ & $6(2.1)$ & $7(3.6)$ & Recessive & $1.65(0.54-5.02)$ & 0.380 \\
\hline & & & & & Overdominant & $0.98(0.67-1.45)$ & 0.930 \\
\hline \multirow[t]{4}{*}{$N G F R$} & rs11466155 & $\mathrm{C} / \mathrm{C}$ & $251(86.8)$ & $178(90.4)$ & Codominant & $0.73(0.42-1.28)$ & 0.260 \\
\hline & Synonymous & $\mathrm{T} / \mathrm{C}$ & $37(12.8)$ & $18(9.1)$ & Dominant & $0.69(0.39-1.24)$ & 0.210 \\
\hline & & $\mathrm{T} / \mathrm{T}$ & $1(0.4)$ & $1(0.5)$ & Recessive & $1.66(0.10-26.89)$ & 0.720 \\
\hline & & & & & Overdominant & $0.67(0.37-1.22)$ & 0.180 \\
\hline
\end{tabular}

*Total numbers of SNPs differ because the genotypes of some SNPs were not determined. 
Table 4. Logistic regression analysis of neurotrophins and their receptor-encoding gene polymorphisms in IgA nephropathy patients with and without nephrotic range proteinuria $\left(>40 \mathrm{mg} / \mathrm{m}^{2} / \mathrm{h}\right)$ after adjustment for gender and age*

\begin{tabular}{|c|c|c|c|c|c|c|c|}
\hline Gene symbol & SNP & Genotype & $\begin{array}{c}\text { Proteinuria } \leq 40 \mathrm{mg} / \mathrm{m}^{2} / \mathrm{h} \\
n=164(\%)\end{array}$ & $\begin{array}{c}\text { Proteinuria }>40 \mathrm{mg} / \mathrm{m}^{2} / \mathrm{h} \\
n=33(\%)\end{array}$ & Model & OR $(95 \% \mathrm{CI})$ & $p$ \\
\hline \multirow[t]{12}{*}{$N G F$} & rs6330 & $\mathrm{C} / \mathrm{C}$ & 109 (66.9) & $21(65.6)$ & Codominant & $1.02(0.49-2.08)$ & 0.970 \\
\hline & Ala273Val & $\mathrm{T} / \mathrm{C}$ & $50(30.7)$ & $10(31.2)$ & Dominant & $0.99(0.44-2.22)$ & 0.970 \\
\hline & & $\mathrm{T} / \mathrm{T}$ & $4(2.5)$ & $1(3.1)$ & Recessive & $1.32(0.14-12.56)$ & 0.810 \\
\hline & & & & & Overdominant & $0.95(0.42-2.18)$ & 0.910 \\
\hline & rs3738701 & $\mathrm{C} / \mathrm{C}$ & $141(86)$ & $32(97)$ & Codominant & $0.16(0.02-1.27)$ & 0.024 \\
\hline & Intron & $\mathrm{A} / \mathrm{C}$ & $22(13.4)$ & $1(3)$ & Dominant & $0.16(0.02-1.25)$ & 0.025 \\
\hline & & $\mathrm{A} / \mathrm{A}$ & $1(0.6)$ & $0(0)$ & Recessive & $0.00(0.00-\mathrm{NA})$ & 0.470 \\
\hline & & & & & Overdominant & $0.17(0.02-1.35)$ & 0.034 \\
\hline & rs11102930 & $\mathrm{C} / \mathrm{C}$ & $58(35.8)$ & 13 (40.6) & Codominant & $0.73(0.41-1.30)$ & 0.280 \\
\hline & Promoter & $\mathrm{T} / \mathrm{C}$ & $78(48.1)$ & $17(53.1)$ & Dominant & $0.86(0.39-1.89)$ & 0.700 \\
\hline & & $\mathrm{T} / \mathrm{T}$ & $26(16.1)$ & $2(6.2)$ & Recessive & $0.32(0.07-1.46)$ & 0.095 \\
\hline & & & & & Overdominant & $1.33(0.61-2.89)$ & 0.470 \\
\hline \multirow[t]{14}{*}{ NTRK1 } & rs926103 & $\mathrm{G} / \mathrm{G}$ & $116(70.7)$ & $21(63.6)$ & Codominant & $0.98(0.47-2.06)$ & 0.970 \\
\hline & Promoter & $\mathrm{A} / \mathrm{G}$ & $43(26.2)$ & $12(36.4)$ & Dominant & $1.13(0.50-2.56)$ & 0.770 \\
\hline & & $\mathrm{A} / \mathrm{A}$ & $5(3)$ & $0(0)$ & Recessive & $0.00(0.00-\mathrm{NA})$ & 0.170 \\
\hline & & & & & Overdominant & $1.31(0.58-2.98)$ & 0.520 \\
\hline & rs2150906 & G & $311(94.8)$ & $59(91.2)$ & & $0.65(0.23-1.82)$ & $1.000 \dagger$ \\
\hline & Promoter & A & $17(5.2)$ & $5(8.8)$ & & & \\
\hline & rs1800601 & $\mathrm{T} / \mathrm{T}$ & $116(70.7)$ & $21(63.6)$ & Codominant & $0.98(0.47-2.06)$ & 0.970 \\
\hline & $5^{\prime}$ UTR & $\mathrm{T} / \mathrm{C}$ & 43 (26.2) & $12(36.4)$ & Dominant & $1.13(0.50-2.56)$ & 0.770 \\
\hline & & $\mathrm{C} / \mathrm{C}$ & $5(3)$ & $0(0)$ & Recessive & $0.00(0.00-\mathrm{NA})$ & 0.170 \\
\hline & & & & & Overdominant & $1.31(0.58-2.98)$ & 0.520 \\
\hline & rs6334 & $\mathrm{G} / \mathrm{G}$ & $71(43.3)$ & $10(30.3)$ & Codominant & $1.22(0.69-2.15)$ & 0.500 \\
\hline & Synonymous & $\mathrm{A} / \mathrm{G}$ & $71(43.3)$ & $21(63.6)$ & Dominant & $1.92(0.84-4.38)$ & 0.110 \\
\hline & & $\mathrm{A} / \mathrm{A}$ & $22(13.4)$ & $2(6.1)$ & Recessive & $0.45(0.10-2.06)$ & 0.260 \\
\hline & & & & & Overdominant & $2.40(1.09-5.28)$ & 0.026 \\
\hline \multirow[t]{8}{*}{ NTRK2 } & rs1187321 & $\mathrm{A} / \mathrm{A}$ & $100(61)$ & $18(54.5)$ & Codominant & $1.07(0.56-2.04)$ & 0.850 \\
\hline & Promoter & $\mathrm{A} / \mathrm{T}$ & $57(34.8)$ & $14(42.4)$ & Dominant & $1.18(0.55-2.56)$ & 0.670 \\
\hline & & $\mathrm{T} / \mathrm{T}$ & $7(4.3)$ & $1(3)$ & Recessive & $0.59(0.07-5.11)$ & 0.620 \\
\hline & & & & & Overdominant & $1.29(0.59-2.82)$ & 0.520 \\
\hline & rs1187323 & $\mathrm{A} / \mathrm{A}$ & $100(61)$ & $18(54.5)$ & Codominant & $1.07(0.56-2.04)$ & 0.850 \\
\hline & Promoter & $\mathrm{A} / \mathrm{C}$ & $57(34.8)$ & $14(42.4)$ & Dominant & $1.18(0.55-2.56)$ & 0.670 \\
\hline & & $\mathrm{C} / \mathrm{C}$ & $7(4.3)$ & $1(3)$ & Recessive & $0.59(0.07-5.11)$ & 0.620 \\
\hline & & & & & Overdominant & $1.29(0.59-2.82)$ & 0.520 \\
\hline \multirow[t]{16}{*}{$B D N F$} & rs11030104 & $\mathrm{A} / \mathrm{A}$ & 39 (23.8) & $12(36.4)$ & Codominant & $0.49(0.28-0.86)$ & 0.010 \\
\hline & Intron & $\mathrm{A} / \mathrm{G}$ & $78(47.6)$ & $19(57.6)$ & Dominant & $0.58(0.26-1.31)$ & 0.200 \\
\hline & & $\mathrm{G} / \mathrm{G}$ & 47 (28.7) & $2(6.1)$ & Recessive & $0.14(0.03-0.63)$ & 0.001 \\
\hline & & & & & Overdominant & $1.70(0.78-3.68)$ & 0.180 \\
\hline & rs7103411 & $\mathrm{T} / \mathrm{T}$ & 39 (23.8) & $12(36.4)$ & Codominant & $0.49(0.28-0.86)$ & 0.010 \\
\hline & Intron & $\mathrm{T} / \mathrm{C}$ & $78(47.6)$ & $19(57.6)$ & Dominant & $0.58(0.26-1.31)$ & 0.200 \\
\hline & & $\mathrm{C} / \mathrm{C}$ & 47 (28.7) & $2(6.1)$ & Recessive & $0.14(0.03-0.63)$ & 0.001 \\
\hline & & & & & Overdominant & $1.70(0.78-3.68)$ & 0.180 \\
\hline & rs7103873 & $\mathrm{G} / \mathrm{G}$ & $52(31.7)$ & $4(12.1)$ & Codominant & $1.72(0.99-2.99)$ & 0.050 \\
\hline & Intron & $\mathrm{G} / \mathrm{C}$ & $80(48.8)$ & $20(60.6)$ & Dominant & $3.56(1.17-10.79)$ & 0.012 \\
\hline & & $\mathrm{C} / \mathrm{C}$ & $32(19.5)$ & $9(27.3)$ & Recessive & $1.41(0.59-3.39)$ & 0.440 \\
\hline & & & & & Overdominant & $1.80(0.82-3.92)$ & 0.140 \\
\hline & rs6484320 & $\mathrm{A} / \mathrm{A}$ & $39(23.8)$ & $12(36.4)$ & Codominant & $0.49(0.28-0.86)$ & 0.010 \\
\hline & Intron & T/A & $78(47.6)$ & $19(57.6)$ & Dominant & $0.58(0.26-1.31)$ & 0.200 \\
\hline & & $\mathrm{T} / \mathrm{T}$ & 47 (28.7) & $2(6.1)$ & Recessive & $0.14(0.03-0.63)$ & 0.001 \\
\hline & & & & & Overdominant & $1.70(0.78-3.68)$ & 0.180 \\
\hline \multirow[t]{4}{*}{ NTRK3 } & rs1128994 & $\mathrm{C} / \mathrm{C}$ & $105(64.4)$ & $19(59.4)$ & Codominant & $1.26(0.65-2.43)$ & 0.500 \\
\hline & Synonymous & $\mathrm{T} / \mathrm{C}$ & $53(32.5)$ & $11(34.4)$ & Dominant & $1.23(0.56-2.70)$ & 0.610 \\
\hline & & $\mathrm{T} / \mathrm{T}$ & $5(3.1)$ & $2(6.2)$ & Recessive & $1.90(0.34-10.62)$ & 0.480 \\
\hline & & & & & Overdominant & $1.10(0.48-2.48)$ & 0.830 \\
\hline \multirow[t]{4}{*}{$N G F R$} & rs11466155 & $\mathrm{C} / \mathrm{C}$ & $149(90.8)$ & $29(87.9)$ & Codominant & $1.46(0.46-4.60)$ & 0.530 \\
\hline & Synonymous & $\mathrm{T} / \mathrm{C}$ & $14(8.5)$ & $4(12.1)$ & Dominant & $1.56(0.47-5.20)$ & 0.480 \\
\hline & & $\mathrm{T} / \mathrm{T}$ & $1(0.6)$ & $0(0)$ & Recessive & $0.00(0.00-\mathrm{NA})$ & 0.690 \\
\hline & & & & & Overdominant & $1.62(0.48-5.43)$ & 0.450 \\
\hline
\end{tabular}

*Total numbers of SNP differ because the genotypes of some SNPs were not determined.

$\dagger$ As the allele frequencies of NTRK1 rs2150906 that did not have all three genotypes present in the study population, only allele frequencies were compared by the Chi-square test. And they were taken into account of Bonferroni's correction. 
velopment in the postinductive stage, and inhibition of NT expression inhibits kidney morphogenesis $(29,30)$. Some recent studies have shown strong expressions of NTs in diseased compared with normal human kidneys, especially for patients with GN and IgAN showed strong expressions of NTRs in the mesangial proliferation areas $(24,31,32)$.

In terms of the renal disease progression, marked expressions of NGF and NGFR are reported in human proteinuric renal diseases (24), and the high levels of NGF expression in monocytes are associated with the presence of proteinuria in patients with GN. Furthermore, limited evidence exists for the involvement of NTs in the fibrotic processes as suggested by our data. NTs are expressed in renal interstitial fibroblasts $(2,32)$ and they are found in the interstitial area near peritubular spaces and in the area of interstitial fibrosis, specifically in patients with IgAN, suggesting that NT systems could be associated with the pathogenesis of IgAN progression (24,31).

Although our data implicate SNPs of NTs and their receptors in the pathophysiology of IgAN, defining the exact function of these SNPs is difficult. In the analysis of our casecontrol study, NGF rs11102930 (promoter) was found to be associated with the susceptibility to childhood IgAN with a sufficient sample power. Moreover, associations between pathogenesis of $\operatorname{IgAN}$ and some candidate SNPs were found in the patient subgroup analysis: $N G F$ rs6330 (missenese), NTRK1 rs6334 (synonymous), BDNF rs11030104 (intron), $B D N F$ rs7103411 (intron), BDNF rs7103873 (intron), and $B D N F$ rs6484320 (intron).

As the missense SNPs are defined to make amino acid sequence changes (Ala to Val in NGF rs6330), they have enough possibilities to affect protein expression and its function. However, it is very difficult to explain the expected effects in the pathogenesis of intron and synonymous SNPs, which do not affect the amino acid sequences. Recently, it has been issued that synonymous SNPs might play an important role in the protein activities and specificities without influencing amino acid sequences $(33,34)$. In addition, some introns have been found to affect various degrees of the efficiency of normal splicing and to structurally stabilize pre-mRNA to protect it against degradation, control the expression of exons (miRNA) to protect from degradation, and enhance protein production $(35,36)$. However, it would be a more reasonable explanation that these SNPs might be linked with other coding SNPs as a haplotype to affect the phenotype in this case.

In terms of promoter SNPs, we investigated whether these genetic variants influence transcription factor binding sites to examine the transcription binding activity of three promoter SNPs (NGF rs11102930, NTRK2 rs1187321, and NTRK2 rs1187323) that showed significant association with the pathogenesis of $\operatorname{IgAN}$. The transcription factor binding sites were compared using the online program "AliBaba 2.1" (http://www.gene-regulation.com/pub/programs/alibaba2). In the NTRK2 rs1187321 site, two transcription factors (HNF-1, NF-ATc3, C/EBPa1p, Id3, and AFP1) can bind to the A-containing sequence, and only two (Oct-2.1 and HNF-1) to the T-containing sequence. In the NGF rs11102930 site, Sp1 can bind to the $\mathrm{C}$, and no transcription factor to the T. However, sequence change in NTRK2 rs1187323 (A to C) did not affect binding of transcription factors (Sp1). This result indicates that NTRK2 rs1187321 and NGF rs11102930 have possibilities to influence protein expressions and/or their functions.

To our knowledge, there has been no report of an association between NT- and NTR-related polymorphisms and patients with GN. However, it is evident that NTs and NTRs could act as an important pathway during the development and progression of IgAN. Thus, we suggest taking a new insight into the neurotrophin pathway considering the pathogenesis of IgAN and it is necessary to investigate more SNPs of NT- and NTR-encoding genes.

In summary, we found that some SNPs of NT- and NTRencoding genes were associated with susceptibility to IgAN and with the pathologic progression of $\operatorname{IgAN}$, namely, the presence of proteinuria, renal disease progression markers, and podocyte foot process effacement.

\section{REFERENCES}

1. Nockher WA, Renz H 2005 Neurotrophins in clinical diagnostics: pathophysiology and laboratory investigation. Clin Chim Acta 352:49-74

2. Micera A, Lambiase A, Stampachiacchiere B, Bonini S, Levi-Schaffer F 2007 Nerve growth factor and tissue repair remodeling: trkA(NGFR) and p75(NTR), two receptors one fate. Cytokine Growth Factor Rev 18:245-256

3. Seidel MF, Herguijuela M, Forkert R, Otten U 2010 Nerve growth factor in rheumatic diseases. Semin Arthritis Rheum 40:109-26

4. Szczepankiewicz A, Rose-Zerilli MJ, Barton SJ, Holgate ST, Holloway JW 2009 Association analysis of brain-derived neurotrophic factor gene polymorphisms in asthmatic families. Int Arch Allergy Immunol 149:343-349

5. Liguori M, Fera F, Patitucci A, Manna I, Condino F, Valentino P, Telarico P, Cerasa A, Gioia MC, di Palma G, Quattrone A 2009 A longitudinal observation of brain-derived neurotrophic factor mRNA levels in patients with relapsing-remitting multiple sclerosis. Brain Res 1256:123-128

6. Hoffjan S, Parwez Q, Petrasch-Parwez E, Stemmler S 2009 Variation in the BDNF and NGFB genes in German atopic dermatitis patients. Mol Cell Probes 23:35-38

7. Szczepankiewicz A, Breborowicz A, Skibinska M, Wilkosc M, Tomaszewska M, Hauser J 2007 Association analysis of brain-derived neurotrophic factor gene polymorphisms in asthmatic children. Pediatr Allergy Immunol 18:293-297

8. Solé X, Guino E, Valls J, Iniesta R, Moreno V 2006 SNPStats: a web tool for the analysis of association studies. Bioinformatics 22:1928-1929

9. Lewis CM 2002 Genetic association studies: design, analysis and interpretation. Brief Bioinform 3:146-153

10. Hedrick PW 1987 Gametic disequilibrium measures: proceed with caution. Genetics 117:331-341

11. Stephens M, Smith NJ, Donnelly P 2001 A new statistical method for haplotype reconstruction from population data. Am J Hum Genet 68:978-989

12. Lee HS, Lee MS, Lee SM, Lee SY, Lee ES, Lee EY, Park SY, Han JS, Kim S, Lee JS 2005 Histological grading of IgA nephropathy predicting renal outcome: revisiting H. S. Lee's glomerular grading system. Nephrol Dial Transplant 20:342-348

13. Gabriel SB, Schaffner SF, Nguyen H, Moore JM, Roy J, Blumenstiel B, Higgins J, DeFelice M, Lochner A, Faggart M, Liu-Cordero SN, Rotimi C, Adeyemo A, Cooper R, Ward R, Lander ES, Daly MJ, Altshuler D 2002 The structure of haplotype blocks in the human genome. Science 296:2225-2229

14. Nassenstein C, Mohring UH, Luttmann W, Virchow JC Jr, Braun A 2006 Differential expression of the neurotrophin receptors p75NTR, TrkA, TrkB and TrkC in human peripheral blood mononuclear cells. Exp Toxicol Pathol 57:55-63

15. Barthel C, Yeremenko N, Jacobs R, Schmidt RE, Bernateck M, Zeidler H, Tak PP, Baeten D, Rihl M 2009 Nerve growth factor and receptor expression in rheumatoid arthritis and spondyloarthritis. Arthritis Res Ther 11:R82

16. Rihl M, Kruithof E, Barthel C, De Keyser F, Veys EM, Zeidler H, Yu DT, Kuipers JG, Baeten D 2005 Involvement of neurotrophins and their receptors in spondyloarthritis synovitis: relation to inflammation and response to treatment. Ann Rheum Dis 64:1542-1549

17. Grimsholm O, Guo Y, Ny T, Forsgren S 2008 Expression patterns of neurotrophins and neurotrophin receptors in articular chondrocytes and inflammatory infiltrates in knee joint arthritis. Cells Tissues Organs 188:299-309

18. Bracci-Laudiero L, Aloe L, Levi-Montalcini R, Galeazzi M, Schilter D, Scully JL, Otten U 1993 Increased levels of NGF in sera of systemic lupus erythematosus patients. Neuroreport 4:563-565

19. Aalto K, Korhonen L, Lahdenne P, Pelkonen P, Lindholm D 2002 Nerve growth factor in serum of children with systemic lupus erythematosus is correlated with disease activity. Cytokine 20:136-139

20. Laudiero LB, Aloe L, Levi-Montalcini R, Buttinelli C, Schilter D, Gillessen S, Otten U 1992 Multiple sclerosis patients express increased levels of beta-nerve growth factor in cerebrospinal fluid. Neurosci Lett 147:9-12

21. Aloe L, Tuveri MA, Carcassi U, Levi-Montalcini R 1992 Nerve growth factor in the synovial fluid of patients with chronic arthritis. Arthritis Rheum 35:351-355 
22. Falcini F, Cerinic MM, Ermini M, Generini S, Lombardi A, Pignone A, Leoncini G, Tirassa P, Aloe L 1996 Nerve growth factor circulating levels are increased in Kawasaki disease: correlation with disease activity and reduced angiotensin converting enzyme levels. J Rheumatol 23:1798-1802

23. Wheeler EF, Gong H, Grimes R, Benoit D, Vazquez L 1998 p75NTR and Trk receptors are expressed in reciprocal patterns in a wide variety of non-neural tissues during rat embryonic development, indicating independent receptor functions. J Comp Neurol 391:407-428

24. Bonofiglio R, Antonucci MT, Papalia T, Romeo F, Capocasale G, Caroleo MC, Di Fausto V, Aloe L 2007 Nerve growth factor (NGF) and NGF-receptor expression in diseased human kidneys. J Nephrol 20:186-195

25. Huber LJ, Hempstead B, Donovan MJ 1996 Neurotrophin and neurotrophin receptors in human fetal kidney. Dev Biol 179:369-381

26. Durbeej M, Soderstrom S, Ebendal T, Birchmeier C, Ekblom P 1993 Differential expression of neurotrophin receptors during renal development. Development 119:977-989

27. Wheeler EF, Bothwell M 1992 Spatiotemporal patterns of expression of NGF and the low-affinity NGF receptor in rat embryos suggest functional roles in tissue morphogenesis and myogenesis. J Neurosci 12:930-945

28. Shibayama E, Koizumi H 1996 Cellular localization of the Trk neurotrophin receptor family in human non-neuronal tissues. Am J Pathol 148:1807-1818
29. Sariola H, Saarma M, Sainio K, Arumae U, Palgi J, Vaahtokari A, Thesleff I, Karavanov A 1991 Dependence of kidney morphogenesis on the expression of nerve growth factor receptor. Science 254:571-573

30. García-Suárez O, Gonzalez-Martinez T, Germana A, Monjil DF, Torrecilla JR, Laura R, Silos-Santiago I, Guate JL, Vega JA 2006 Expression of TrkB in the murine kidney. Microsc Res Tech 69:1014-1020

31. Antonucci MT, Bonofiglio R, Papalia T, Caruso F, Caroleo MC, Mancuso D, Aloe L 2009 Nerve growth factor and its monocyte receptors are affected in kidney disease. Nephron Clin Pract 111:c21-c28

32. Alpers CE, Hudkins KL, Ferguson M, Johnson RJ, Schatteman GC, Bothwell M 1993 Nerve growth factor receptor expression in fetal, mature, and diseased human kidneys. Lab Invest 69:703-713

33. Kimchi-Sarfaty C, Oh JM, Kim IW, Sauna ZE, Calcagno AM, Ambudkar SV, Gottesman MM 2007 A "silent" polymorphism in the MDR1 gene changes substrate specificity. Science 315:525-528

34. Komar AA 2007 Silent SNPs: impact on gene function and phenotype. Pharmacogenomics 8:1075-1080

35. Malisic EJ, Jankovic RN, Radulovic SS 2010 An intronic variant in the TP53 gene in Serbian women with cervical or ovarian cancer. Cancer Genet Cytogenet 198:173-175

36. Ying SY, Chang CP, Lin SL 2010 Intron-mediated RNA interference, intronic microRNAs, and applications. Methods Mol Biol 629:205-237 